\title{
MedienPädagogik
}

Zeitschrift für Theorie und Praxis der Medienbildung

Themenheft Nr. 41: Inklusiv-mediale Bildung in schulischen Kontexten. Eine interdisziplinäre Bestandsaufnahme

Herausgegeben von Christian Filk und Heike Schaumburg

\section{Spielend soziale Inklusion fördern}

\section{Zur aktiven Auseinandersetzung mit sozialen Differenzlinien durch game- based Learning Activities}

Lisa-Katharina Möhlen, Daniel Handle-Pfeiffer, Alexander Schmoelz, Michelle Proyer und Gertraud Kremsner

\section{Zusammenfassung}

Dieser Beitrag beleuchtet, wie spielerische Pädagogik Inklusion befördern kann. Dazu wurden Spieleworkshops in (ausser-)schulischen Kontexten durchgeführt. Diese wurden methodisch mit diversen qualitativen Erhebungsinstrumenten begleitet und mittels der Dokumentarischen Methode ausgewertet. Die Analyse der erhobenen Daten zeigt, wie sich die Spielenden mit sozialen Differenzlinien wie Be-hinderung und Gender während des Spielens und in post game-based activities auseinandersetzen. Ausgehend davon sollen inklusionsfördernde Potentiale in spielerischer Pädagogik identifiziert werden. Es werden drei inklusionsbezogene Kompetenzen als Analysemerkmal verwendet: Reflektierende Debatte, Kreatives Denken und Konfliktlösungsstrategien. Zentrale Ergebnisse sind, dass Spielende im Spielgeschehen mit sozialen Differenzen konfrontiert werden. Dahingehend wird in den post game-based activities die Reflexion über Handlungen und Interaktionen in Bezug auf soziale Unterschiede forciert.

Fostering Social Inclusion through Playful Pedagogy

\begin{abstract}
The following paper shows how inclusion can be fostered through playful pedagogy. The Erasmus+ project 'eCrisis' is the initial point of this research. For the data collection, workshops in school and other educational institutions were held. Data were collected by various qualitative methods and evaluated by the Documentary Method. Results show that the core focus lies on players' interaction and the handling of social inequalities. Those aspects are the main base to identify inclusive potentials while playing and post game-based activities. Therefore, three competences are set to analyse the data: reflective debating, creative thinking and conflict resolution. The most important outcomes are that players are confronted with social inequality during playing. The interaction and actions are determined by habitualised and spontaneous structures. On the other side, reflective processes regarding social inequalities are happening during post game-activities.
\end{abstract}




\section{Einleitung}

In Zeiten gesellschaftlichen Wandels, in welchen die Schere zwischen Arm und Reich weiter auseinander zu driften droht sowie Populismus und rechtskonservative Mächte sich im Aufwind befinden, sind gerade marginalisierte Personen(-gruppen) wie z. B. Menschen mit Migrations- und Fluchthintergrund, aber auch Menschen mit Behinderungen ${ }^{1}$ verstärkt von sozialer Exklusion betroffen. Auch Frauen werden als soziale Gruppe verstärkt in stereotype Rollenbilder gedrängt (Biewer, Proyer, und Kremsner 2019).

Ausserdem ist die Gesellschaft mit einer voranschreitenden Digitalisierung konfrontiert, welche einen digitial divide hervorrufen kann (vgl. Iske und Verständig 2014). Um assoziierte gesellschaftliche Zusammenhänge aktiv und partizipativ zu erforschen, wurde das Erasmus+ Projekt eCrisis erfolgreich beantragt (eCrisis 2018). Das konkrete Ziel des dreijährigen eCrisis-Projektes ist es, Lehrende dazu zu befähigen, inklusive Strukturen im Unterricht mittels des Konzepts des (digital) gamebased learnings zu etablieren. Auf diese Weise können Schülerinnen und Schüler über das vertraute Element des Spielens menschliche Vielfalt und Inklusion erfahren und verstehen lernen.

Die Analyse des Ist-Standes in den teilnehmenden Ländern Griechenland, Malta und Österreich ergab, dass Exklusionsprozesse besonders deutlich in den kategorialen Zuschreibungen Be-hinderung, Migration und Gender zu erkennen sind. Um diesen Entwicklungen entgegenzuwirken, wurden pädagogische Szenarien entwickelt, in welchen Spiele als Methode angewendet werden, um Inklusion in europäischen Klassenzimmern zu fördern (vgl. Schmoelz et al. 2017). In Österreich wurden 13 Spieleworkshops mit über 270 Teilnehmenden verschiedener Institutionen abgehalten. Zum Einsatz kamen sowohl analoge als auch digitale Spiele; bei Letzteren lag der Fokus im Einklang mit den Zielen des Projektes auf dem Spiel Village Voices (vgl. Yannakakis et al. 2010). Es wurde anhand dieser Best Practice-Beispiele herausgearbeitet, dass der Einsatz von digitalen Spielen in Kombination mit post game-based activities - also der Spielsequenz nachgeordneten Aktivitäten wie z. B. game-based dialogues - Platz für Reflexionen des sozialen Umgangs im Klassenraum, aber auch aktueller gesellschaftlicher Themen und Problemlagen bietet. Der hier vorliegende Beitrag basiert auf diesen Erkenntnissen.

Um Inklusionsprozesse mittels (digitaler) Spielen noch gezielter stärken zu können, muss zusätzlich analysiert werden, wie die teilnehmenden Spielenden in den Spielsituationen mit auftretenden sozialen Differenzen umgehen. Demnach konnten folgende Forschungsfragen formuliert werden:

1 Behinderung wird im Diskurs der Disability Studies nicht als medizinische Defizitzuschreibung gesehen, sondern vielmehr als Produkt sozialer Konstruktionen, welche auf gesellschaftlichen Machtverhältnissen und Hierarchien fussen (vgl. Waldschmidt 2005, 18). 
- Wie setzen sich Spielende während des Spielprozesses von digitalen und analogen Spielen sowie daran anschliessenden post game-based activities mit sozialen Differenzlinien auseinander?

- Inwiefern kann durch Verknüpfung von game-based learning und post gamebased activities soziale Inklusion gefördert werden?

\section{Spielerische Pädagogik für Inklusion}

\subsection{Inklusion}

In Anlehnung an Fuchs $(2016,389)$ wird Inklusion - wie in der bereits zuvor bestehenden und im Rahmen des Projektes modifizierten Abbildung 1 zu sehen - in Abgrenzung zu Integration und Exklusion verstanden. Das Modell ist sowohl auf gesamtgesellschaftlicher Makroebene als auch auf systembasierter Mesoebene (wie zum Beispiel dem Schulkontext) anwendbar.

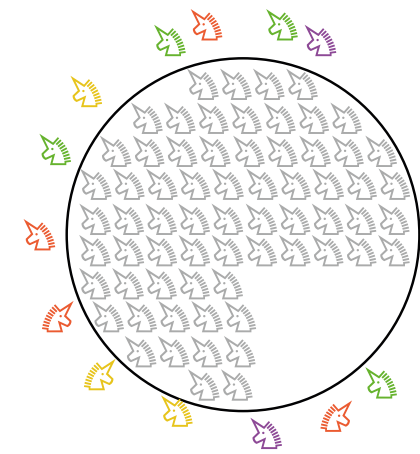

Exklusion

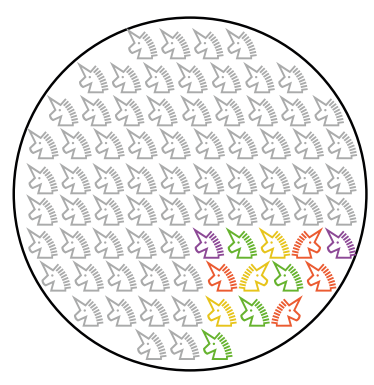

Integration

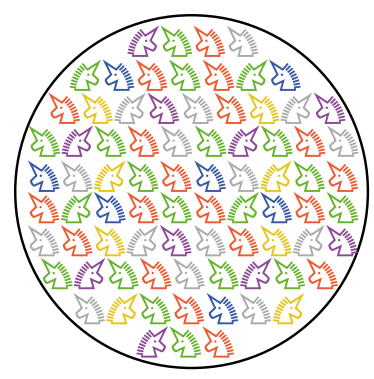

Inklusion

Abb. 1.: Exklusion-Integration-Inklusions-Schema (Schmoelz et al. 2017, 4)

Auf der linken Seite der Abbildung ist das Konzept der Exklusion illustriert. Es wird ersichtlich, dass einzelne Individuen ausgeschlossen werden und keine Möglichkeit zur Partizipation in und an der Mehrheitsgesellschaft erhalten (vgl. ebd., 399). In der Mitte ist das Modell der Integration veranschaulicht. Einzelne Individuen können teilhaben, allerdings unter Vorbehalt anpassungsbedingter Massnahmen, wie zum Beispiel Therapien oder weiterer Fördermassnahmen (Hillmann 1994, 377). Dies zielt darauf ab, einzelne Individuen an die normativen Rahmenbedingungen der Mehrheitsgesellschaft anzupassen. Somit ist auch innerhalb eines integrativen Verständnisses ein exkludierendes Moment enthalten (vgl. Hinz 2002, 359). 
Die Europäische Kommission (2016) definiert Inklusion wie folgt: «active inclusion means enabling every citizen, notably the most disadavantaged, to fully participate in society, including having a job.» Inklusion wird hier vorwiegend als arbeitsmarktbedingte Partizipation definiert, wobei Beschäftigung als wesentlicher Faktor gesehen wird, um Armut und daraus resultierend das Risiko gesellschaftlicher Exklusion zu verhindern. Diese Definition bezieht sich somit nicht auf alle Lebensbereiche, sodass eine Erweiterung bzw. Ergänzung der Definition notwendig ist, um zu einem breiteren Inklusionsverständnis zu gelangen. Hierfür kann eine Definition der UNESCO (2005) eingebracht werden, welche Inklusion als Streben nach vollkommener Partizipation auf allen Ebenen ansieht, sodass jeder Person der Zugang zu Bildung und im weiteren Sinne volle gesellschaftliche Teilhabe ermöglicht wird.

Dieses weite Verständnis von Inklusion liegt dem Projekt zugrunde; davon ausgehend wird das Anstossen von Empowermentprozessen intendiert. Erreicht werden soll dies, indem drei zentrale Kernprozesse zum Einsatz kommen: Inklusion soll mittels (1) reflektierender Diskussion, (2) kreativem Denken und (3) Konfliktlösungsstrategien erlernt und in weiterer Folge umgesetzt werden.

\section{Reflektierende Diskussion}

Der erste Prozess besteht aus zwei Elementen. Reflexion bedeutet, dass sich Schülerinnen und Schüler in systematischer Weise mit einem Gegenstand oder einer Situation auseinandersetzen (vgl. Miettinen 2000). Dafür gilt es, bestimmte vorgefertigte Denkweisen zu hinterfragen sowie aufkommende Probleme oder Fragen (neu) zu betrachten. Diskussion oder Debatte als Begriff wiederum impliziert, dass sich Schülerinnen und Schüler über jene Reflexionsprozesse in angemessener, wertschätzender und fairer Weise austauschen. Dabei geht es - Dewey $(1933,9)$ folgend - um eine «active, persistent, and careful consideration of any belief or supposed form of knowledge in the light of the grounds that support it». Davon ausgehend, so Dewey weiter, werden Schülerinnen und Schüler zu folgendem Ziel befähigt: «to transform a situation in which there is experienced obscurity, doubt, conflict, disturbance of some sort, into a situation that is clear, coherent, settled, harmonious» (ebd., 100).

\section{Kreatives Denken}

Kreatives Denken ist eine Möglichkeit, sozialen Interaktionen und Differenzen zu begegnen. Es gibt nicht immer nur einen Lösungsweg, um Situationen zu gestalten (vgl. de Bono 1970). Vielmehr geht es darum, die Situation multidimensional zu betrachten und kreative Methoden zu entwickeln, um menschliches Miteinander partizipativ und wertschätzend zu gestalten (ebd.). Aktuelle Forschungsergebnisse belegen, dass kreatives Denken kollaborativ und kommunal eingebettet ist. Kreatives Denken wird durch disruptive Elemente wie Wörter, Symbole und Emotionen angestossen und im Dialog vollzogen (vgl. Stenning et. al. 2016; Schmoelz 2018). 


\section{Konfliktlösungsstrategien}

Konflikte treten in jeglichen zwischenmenschlichen Interaktionen auf. Gerade im Schulalltag kommt es bei Heranwachsenden immer wieder zu Konflikten, welche sich zum Beispiel in Form von Diskussionen, Streit, aber auch Mobbing und Diskriminierung ausdrücken. Durch zunehmende Heterogenität im Klassenraum können soziale Konflikte geschürt werden, welche es zu lösen gilt (vgl. Husband 2007). TingToomy und Oetze (2001) arbeiten heraus, dass sich Schülerinnen und Schüler bereits in der Grundschule mit Konfliktvermeidungs- bzw. -bewältigungsstrategien auseinandersetzen sollten.

Voida et al. (2009) postulieren, dass die drei genannten zentralen Prozesse Reflektierende Diskussion, Kreatives Denken und Konfliktlösungsstrategien mittels (digitalen) Spielen vermittelt werden können, um inklusive Strategien und Prozesse zu fördern.

\subsection{Spielerische Pädagogik}

Spielerische Pädagogik ist «das Anleiten und Begleiten von Kindern durch die transparente pädagogische Einbettung von spielerischen Elementen, digitalen und analogen Lernspielen und spielbasierten Dialogen in den Unterricht und in andere Lehr/Lernsituationen» (Schmoelz 2016, 115). Unter «Spielerischer Pädagogik» werden zentrale didaktische Leitkonzepte - darunter auch game-based learning und gamebased dialogue als post game-based activity als zentrale Leitkonzepte im Rahmen des eCrisis-Projektes - subsumiert. Sie bilden die Basis, um die im Verlauf des eCrisisProjektes generierten pädagogischen Szenarien sowie die verwendeten (digitalen) Spiele zu erläutern. Abschliessend wird dargestellt, inwiefern spielerische Pädagogik (in der spezifischen didaktischen und technologischen Ausformung des eCrisis-Projektes) Inklusion fördern kann.

\subsection{Game-based learning und game-based activities}

Prensky (2001) entwickelte den Begriff des (digital) game-based learning mit der Kernidee, dass Lernen durch Spass und Engagement gefördert werden kann und soll. Spiele werden hier zum Zweck des Vermittelns von Fähigkeiten und Fertigkeiten sowie der Erhöhung von (Lern-)Engagement eingesetzt. Es wird auch als Spielen mit ernsthaften Absichten (vgl. Le, Weber, und Ebner 2013) bezeichnet - ebenso, wie dies bei serious games (vgl. Michael und Chen 2011) der Fall ist. Ebner und Schön (2011) halten fest, dass zwischen beiden Begriffen wenig bzw. kein Unterschied besteht. Für game-based learning werden serious games (im Unterschied zu popular games 
wie etwa Minecraft u.a.) explizit für das Lernen gestaltet; die Unterhaltung ist somit zweitrangig. (Digitale) Spiele werden für didaktische Zwecke im Unterricht eingesetzt; am Ende des Spiels steht die Erreichung eines Lernziels.

Eine didaktische Weiterentwicklung von game-based learning ist in didaktisch ausgearbeiteten post game-based activities zu finden: Schmoelz et al. (2017) entwickelten game-based dialogues, durch welche eine Reflexion der Handlungen und Interaktionen, aber auch der aufgekommenen Gefühle und Bedürfnisse im vorangestellten Spiel ermöglicht und folglich in den Vordergrund gestellt werden. Die abschliessende Reflexion kann z. B. in sokratisch-narrativen oder mit bildbasierten Dialogen (vgl. Schmoelz et al. 2017) umgesetzt werden.

\subsection{Verwendete Spiele}

Die vorgestellten Szenarien wurden anhand von verschiedenen digitalen Spielen, aber auch konventionellen Gesellschaftsspielen (wie Schach, Mensch Ärgere Dich Nicht, Uno, Mikado) erprobt; im Zentrum des Interesses für das eCrisis-Projekt steht aber vor allem das digitale Spiel Village Voices.

Village Voices ist ein Multi-Player-Game für den Computer, das von den griechischen und maltesischen Projektpartnerinnen und -partnern zum Zweck der Förderung sozialer Inklusion entwickelt wurde (vgl. Yannakakis et al. 2010). Es kann gleichzeitig in den Sprachen Deutsch, Englisch und Griechisch gespielt werden. Die Spielwelt wird durch eine schwebende Insel präsentiert, auf der eine Dorfgemeinschaft angesiedelt ist. Diese Gemeinschaft besteht aus vier Charakteren, welche nach Auswahl sowohl durch eine männliche als auch weibliche Spielfigur dargestellt werden können; die Auswahl weiterer Geschlechtsidentitäten ist leider nicht möglich, sodass hier (leider) ausschliesslich dichotome Geschlechterverhältnisse reproduziert werden. Die Rollen - Gastwirt oder Gastwirtin, Heiler oder Heilerin, Schmied oder Schmiedin und Tischler oder Tischlerin - werden vom Computer per Zufallsgenerator vergeben. Jeder Charakter hat berufsspezifische Aufgaben zu erfüllen, welche inm durch Quests angezeigt werden. 


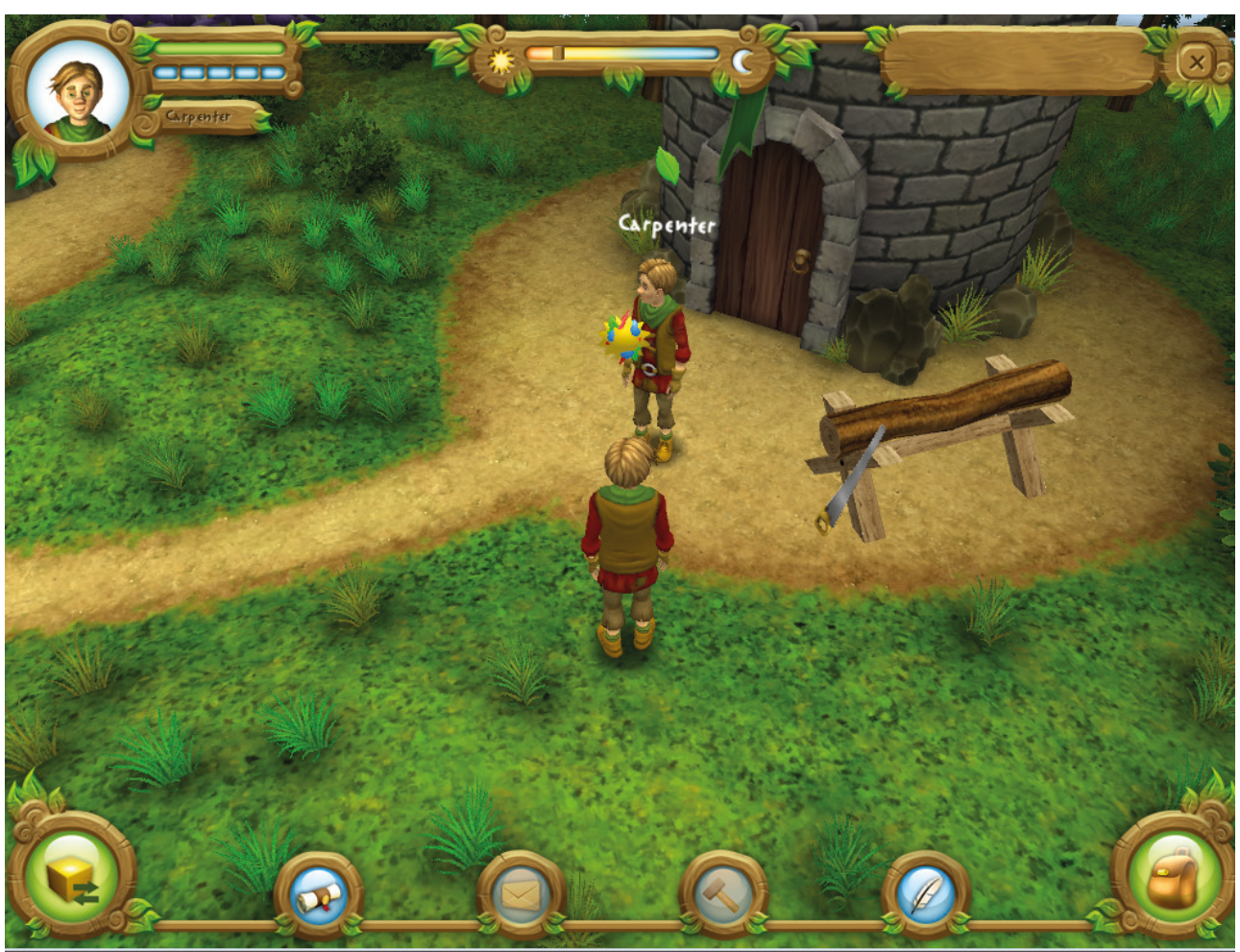

Abb. 1.: Screenshot aus dem Spiel Village Voices.

Die Spielenden können mit ihren produzierten Gütern auch handeln, um Produkte zu erwerben, die sie selbst nicht herstellen können. Zudem müssen sie für ihre Spiel-Charaktere auch menschliche Bedürfnisse wie Schlafen und Essen abdecken. Je mehr Levels absolviert werden, desto mehr sind die Quests darauf ausgelegt, dass die Charaktere miteinander kooperieren müssen, um das nächste Level zu erreichen. Dadurch wird die Abhängigkeit der Charaktere voneinander immer grösser und implizit steigt auch die Konfliktfähigkeit an (vgl. Yannakakis et al. 2010). Zusätzlich ausgelöst werden konfliktbehaftete Situationen durch das Spieldesign, da den Spielenden die Möglichkeit geboten wird, andere Charaktere zu bestehlen oder deren Häuser zu zerstören. Allerdings werden während des Spiels auch inhärente Reflexionstools angeboten, bei denen auf Basis einer Bewertungsskala gefragt wird, wie sich die Spielerinnen und Spielern fühlen (vgl. Cheong et al. 2015). Diese Reflexion geschieht immer nach einem erfolgten Handel, aber auch dann, wenn es zu Diebstahl oder Zerstörung kommt. Das Ziel des Spiels ist es, die Ambivalenz zwischen der Förderung von zwischenmenschlichen Beziehungen im Dorf, dem gemeinsamen Lösen der einzelnen Spiellevels, aber auch dem eigenen Wohlergehen auszubalancieren (vgl. Schmoelz et al. 2017). Künstlich herbeigeführte Konflikte führen dazu, dass sich die Spielenden mit Problematiken in zwischenmenschlicher Interaktion auseinandersetzen müssen, 
da sie ansonsten das Spielziel nicht erreichen. Durch die Reflexionstools setzen sich Spielenden dezidiert mit ihren Gefühlen und Handlungen auseinander. Zudem werden über diese Tools auch Interaktionen bewertet und dadurch eine Reflexion der Handlungen und Aktivitäten der anderen Spielenden erzwungen.

\section{Methodische Vorgehensweise mit der Dokumentarischen Methode}

Für die empirische Bearbeitung der Forschungsfragen wurde die Dokumentarische Methode nach Bohnsack ausgewählt (vgl. Bohnsack, Fritzsche, und Wagner-Willi 2014), um das in den Workshops gesammelte Datenmaterial auszuwerten. Die Methode verfolgt das Ziel, latente Sinn- und Habitusstrukturen der Spielenden zu identifizieren. Es wird nicht davon ausgegangen, dass Interpretationen immer an «verallgemeinerbare Wissensbestände und Normen» geknüpft sind (Bohnsack 2003, 551), denn Interpretationen basieren nicht auf der Abweichung von normativen Bedingungen des oder der Interpretierenden. Somit wird nicht nach den Ursachen der interpretierten Nicht-Normalität gesucht, sondern vielmehr nach der Herstellung, Konstruktion und Konstitution der zu interpretierenden Phänomene. Die Analyse des subjektiven Sinnes bleibt zwar Gegenstand der Interpretationen, bildet jedoch nicht die Grundlage einer wissenschaftlichen Methode. Wissenschaftlichkeit definiert sich in der Dokumentarischen Methode dadurch, dass der bewusste Unterschied von konjunktivem und kommunikativem Wissen deutlich wird (ebd., 561). Durch das Aufzeigen dieses Unterschiedes sollen die Prämissen alltäglichen Wissens methodologisch sowie forschungspraktisch sichtbar gemacht und in einem weiteren Schritt aufgelöst werden (ebd., 559). Sobald die handlungs- und erlebnisbezogene Herstellung der Wirklichkeit Gegenstand der Analyse ist, gelangen die beteiligten Personen auf die Ebene des sogenannten konjunktiven Wissensschatzes (ebd., 562). Dieser drückt sich durch kollektive Sozialisations- und Erfahrungsgrundlagen aus. Somit ist es mittels der Dokumentarischen Methode als Analysestrategie möglich, eine Brücke zwischen konstruktivistischen und objektiven sowie subjektiven und objektiven Forschungspositionen zu ziehen (ebd., 563). Zusammenfassend liegt die Begründung der Dokumentarischen Methode darin, dass die beobachtende Perspektive des oder der Interpretierenden

- die Differenzierung der Sinnstruktur des zu beobachtenden Handelns und den von den Handelnden subjektiv gemeinten Sinn berücksichtigt und

- das Wissen des oder der Handelnden als empirische Basis der Analyse verwendet wird, damit konjunktives Wissen zustande kommen kann (ebd., 560). 


\subsection{Sample und Beobachtungssituation}

In Anschluss an Przyborski und Wohlrab-Sahr (2009) soll eine möglichst heterogene Gruppe akquiriert werden, damit im Fall des Forschungsvorhabens die Zusammensetzung der Spielenden die Dimensionen Be-hinderung, Migration und Gender abdeckt. Es wurden Einladungen an verschiedene Trägerinstitutionen ausgesendet, um dieses Anliegen umzusetzen. Für den ersten Workshop fanden sich 19 Teilnehmende aus unterschiedlichen Kontexten ein. Die Namen der Teilnehmenden wurden zur Gewährleistung ihrer persönlichen Rechte pseudonymisiert. Teilnehmende des ersten Workshops waren: Amir, Anja, Christopher, Derya, Diana, Helga, James, Laura, Linda, Lukas, Maja, Markus, Martina, Mohammad, Ornella, Peter, Sabine, Stella und $\mathrm{Tom}^{2}$. Es standen verschiedene digitale und analoge Spiele zur freien Verfügung. Drei Forschende sassen - selbstverständlich nach vorheriger Abklärung mit den Teilnehmenden sowie deren explizitem Einverständnis - für eine verdeckte Beobachtung hinter einer Spiegelwand, welche von den Workshopteilnehmenden nicht einsehbar war. Die Spielsituationen wurden mit Videokameras aufgezeichnet. Zudem wurden im Raum Tablets als Aufnahmegeräte verteilt, um die einzelnen Gespräche gezielter aufnehmen zu können. Ausserdem waren ein bis zwei Forschende für teilnehmende Beobachtungen direkt im Raum anwesend. Von Seiten des Forschungsteams gab es keinerlei Rahmenbedingungen für die Zusammensetzung der Spielgruppen, einer möglichen zeitlichen Begrenzung oder der Auswahl der Spiele; die Teilnehmenden wählten all dies freiwillig. Insgesamt wurde eineinhalb Stunden gespielt und im Anschluss erfolgte eine halbstündige Reflexionsphase im Plenum. Das Ziel dieser Phase war es zunächst, die Konstitution von Spielgruppen und die dazugehörigen Parameter zu erschliessen.

Um die spielerischen Interaktionen detaillierter analysieren zu können, wurde in einem zweiten Workshop ausschliesslich das Spiel Village Voices gespielt. Entsprechend der Spielvorgaben - spielen können und müssen exakt vier Personen - bestand die zweite Gruppe aus den vier Teilnehmenden Diana, Ludwig, Mustafa und Yussuf. Kontrastierend zu der ersten Gruppe können in einem kleineren Setting die Interaktionen expliziter und detaillierter herausgearbeitet werden. Auch diese Gruppe weist einen sehr heterogenen Charakter auf, da verschiedene Differenzdimensionen wie Be-hinderung, Migration und Gender vertreten sind.

\section{Ergebnisse}

In der Analyse des Datenmaterials zeigten sich mehrere Differenzlinien. Auf Basis der Häufigkeit der im empirischen Material auftretenden Aspekte haben sich zwei Differenzdimensionen als zentral erwiesen: Be-hinderung(en) und Gender. Exemplarisch

2 Bei der Pseudonymisierung wurde darauf geachtet, dass sich die Namenswahl an der sprachlichen Herkunft des Ursprungsnamens orientierte. 
werden im Folgenden Fallbeispiele erläutert, welche eine hohe inhaltliche Generalisierbarkeit für mehrfach vorgefundene Interaktions- und Handlungsweisen aufweisen.

\subsection{Be-hinderung(en) während des Spielens}

Das folgende Beispiel zeigt eine Situation, in welcher das Kartenspiel UNO gespielt wird. Nach mehreren Spielrunden hat James so viele Karten, dass er sie nicht mehr alle halten kann. Anhand des folgenden Ausschnitts wird aufgezeigt, dass das Spiel die Aufnahme von vielen Karten erfordert, welches wiederum James' motorische Fähigkeiten übersteigt. Durch die Vorgaben des Spiels wird James somit be-hindert, die Karten in der Hand zu halten.

Tom: Wir helfen helfen vielleicht beim Kartenhalten

[...]

James: Oder haben wir $(2 \mathrm{sec})$ ein ein ein ein Spielekarton, was man umlegen kann, wo man es dann darauf

Tom: $\quad$ So aufstellen kann

James: Haben wir einen Spielekarton, einen leeren, was man umlegen kann? $[\ldots]$

Lukas: So einen Deckel vielleicht oder was für die Karten

James: Ja, da da unter der Dings, wo es nur reinstecken braucht

Lukas: Ach so reinstecken

James: Also, wo ein ein Karton mit Boden und [...] das muss ich nur dann reinstecken brauch

Tom bietet James seine Unterstützung an, die Karten anstelle von James zu halten. Daraufhin versucht James selbst eine Lösung zu finden, um die Karten ohne Toms Unterstützung zu halten. James scheint auch eine konkrete Idee im Kopf zu haben, wie er das Problem lösen kann. Diesen Gedanken elaboriert er gemeinsam mit seinen Mitspielenden, sodass schlussendlich eine Konstruktion zum Kartenhalten entsteht, welche es James ermöglicht, ohne die Unterstützung einer weiteren spielenden Person weiterhin mitspielen zu können. An dieser Situation ist zu erkennen, dass das Spiel eine Dilemmasituation auslöst, welche zu einer Exklusion der spielenden Person führen kann. Damit dies nicht eintritt, bietet ein Mitspieler James zunächst seine Hilfe an. Durch Kreativität und Kollaboration des Spielteams wird eine Lösungsstrategie herausgearbeitet, sodass James weiterhin ohne die Hilfe von Anderen mitspielen kann.

In der folgenden Spielsituation wird das Computerspiel Village Voices gespielt. Die vier Spielenden sind Diana, Ludwig, Mustafa und Yussuf. Um den Spielverlauf voranzubringen, ist es nötig, dass die Spielenden ihre eigenen Ressourcen untereinander 
tauschen und handeln. Mustafa teilt der Gruppe mit, dass er Holz benötigt. Da Diana die Tischlerin ist, wäre es ihre Aufgabe, Mustafa zu antworten. Da sie dies nicht tut, übernimmt Ludwig die Aufgabe und beantwortet Mustafas Frage. Diana hingegen ist damit beschäftigt, ihren eigenen Bedürfnissen nachzugehen.

Mustafa: Wer hat Holz für mich? (7sec)

Ludwig: Tischlerin! (15sec)

Diana: Wie bekomme ich Energie? (10 sec) Muss ich jetzt in den Wald gehen? Ludwig steht auf und stellt sich neben Diana.

Ludwig: Handeln. Klick auf die Papierrolle. Klicke okay. Jetzt ist die Aufgabe erledigt. (14 sec) Haben wir uns gestritten? Ich glaube nicht (lachend).

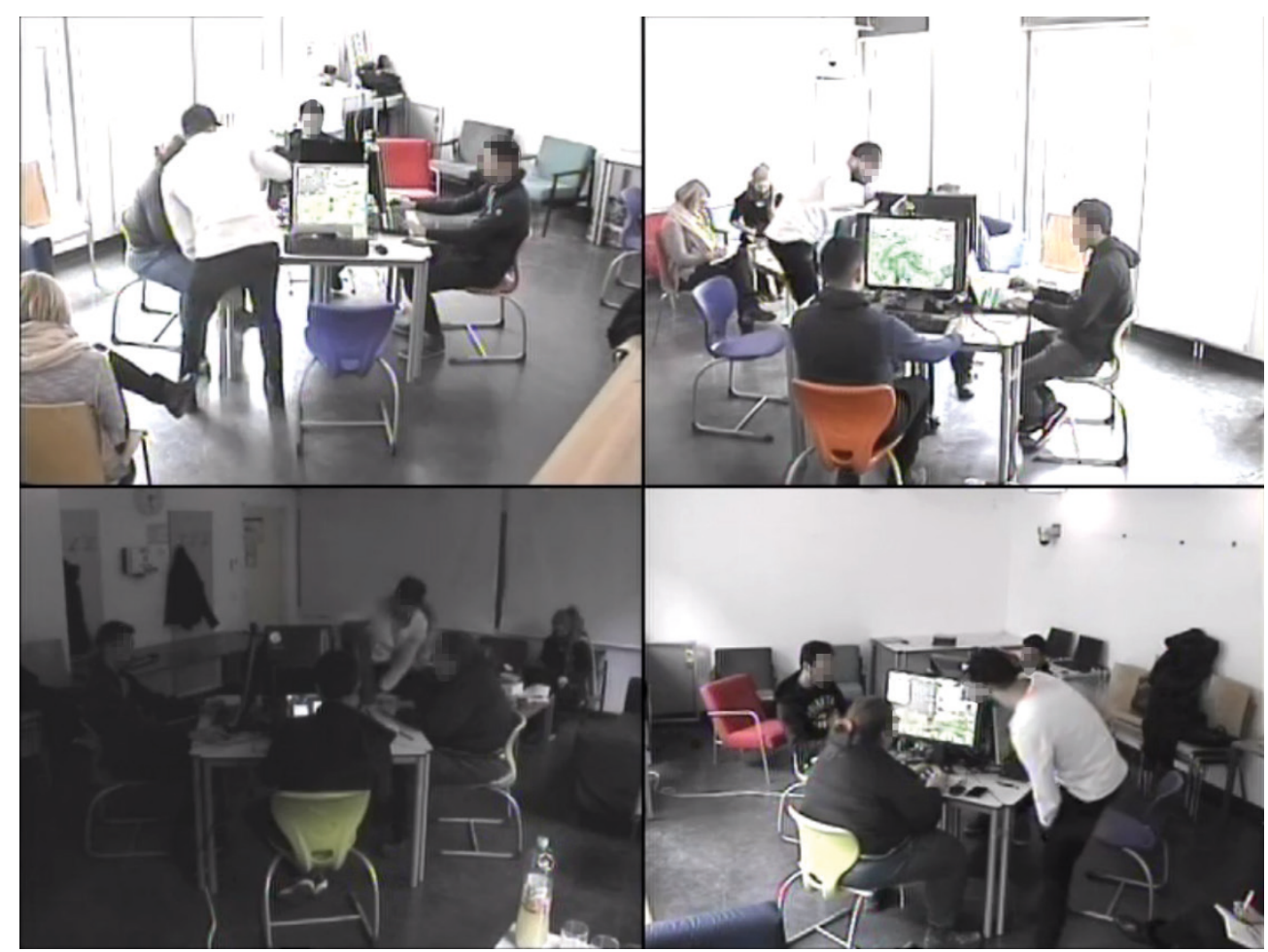

Abb. 1.: Spielsituation Village Voices, Screenshot aus dem Videodatenmaterial. (Ludwig wechselt physisch seinen Platz, um Diana die Spielfunktionen und ihre Aufgaben zu erklären. Dies lässt die Interpretation zu, dass Ludwig die Position von Diana einnimmt, um einen effizienteren Spielverlauf zu ermöglichen. Da Mustafa bereits nach benötigten Ressourcen gefragt hat, aber Diana nicht antwortet, wird der Spielverlauf für 21 Sekunden behindert.) 


\subsection{Gender: Aufbrechen klassischer Geschlechterrollen}

Das erste Beispiel in dieser Differenzdimension zeigt die Eingangssequenz im Spiel Village Voices. Das Spieldesign ermöglicht es, die Spielfiguren sowohl als männliche als auch als weibliche Charaktere darzustellen; andere Geschlechtsidentitäten zu wählen ist leider nicht möglich. Allerdings erscheinen zu Beginn des Spiels vier männliche Dorfbewohner; Spielende müssen, sofern ihnen das wichtig ist, das Geschlecht/Gender der eigenen Spielfigur manuell umstellen. Alle vier behalten die männliche Version bei. Des Weiteren müssen die vier Spielenden herausfinden, welche Rolle sie während des Spiels übernehmen.
Ludwig: Wer ist der Schmied?
Diana: Ich glaube ich
Ludwig: Du bist der Tischler, glaube ich.
Diana: Nein, ich bin die Tischlerin.

Anhand der Sequenz wird deutlich, dass Diana trotz des männlichen Geschlechts ihrer Spielfigur Ludwig in seiner Verwendung der männlichen Form verbessert. Die Identifizierung der einzelnen Rolle und das Spielgeschehen werden weiter fortgesetzt.

Mustafa: Wer hat Holz für mich?

Ludwig: Tischlerin!

Nach Mustafas Frage, wer Holz für inn zum Handeln habe, gibt es von Diana (der Tischlerin) keine Reaktion (s.o.). Daraufhin interveniert Ludwig und weist darauf hin, dass die Tischlerin (Diana) Holz besitzt. Hier ist zu erkennen, dass Ludwig, welcher zuvor das Maskulinum verwendet hat, nun die weibliche Version des Wortes verwendet. Anzumerken ist, dass die Spielcharaktere nach wie vor durch vier männliche Figuren repräsentiert werden.

Eine sehr ähnliche Situation ist in der Einführung zum grossen, d.h. von 19 Teilnehmenden besuchten Spieleworkshop vorzufinden. Eine Person der Forschenden stellt zunächst die Rahmenbedingungen des Workshops vor, um dann detaillierter auf den genauen Ablauf einzugehen.

Forscherin: [...]

Die Linda ist eher für die Brettspiele zuständig (James lacht) und für alle Spiele, die man halt nicht digital spielt (Forscherin lacht) und Christopher ist eher zuständig für die eben digitalen Spiele also für Tablet und für die Computer und so ähm wobei ihr könnt sie natürlich beide alles fragen (Forscherin lacht). 
Wie im ersten Beispiel lässt sich auch hier erkennen, dass den Forschenden zunächst traditionelle und mitunter klischeebesetzte Rollen und Aufgaben zugeschrieben werden, diese aber in einem zweiten Schritt wieder aufgelöst werden. Kontrastierend zum ersten Beispiel verbessert sich die Forscherin revidierend. Es liegt die Vermutung nahe, dass die Forscherin durch das Lachen von James irritiert wurde, welches wiederum darauf hinweisen könnte, dass ihre Aussage eine stereotypische Zuschreibung von geschlechtsspezifischen Kompetenzen impliziert. Im ersten Fall hingegen wird Ludwig durch seine Mitspielerin verbal darauf aufmerksam gemacht, dass er sie mit der männlichen Verwendungsform anspricht. Auch hier kommt es zu einer Veränderung seiner Kommunikationsweise, welche jedoch eine explizite Intervention seiner Mitspielerin erforderte. Festgehalten werden kann demzufolge, dass bei beiden Situationen eine Veränderung zu erkennen ist, welche jedoch durch unterschiedlich intensive Interventionen herbeigeführt wurde.

\section{Diskussion: Differenzlinien überwinden und Inklusion fördern mit spielerischer Pädagogik?}

In der Auswertung des empirischen Datenmaterials und unter Berücksichtigung der zwei Forschungsfragen konnten zwei übergeordnete Aspekte identifiziert werden: das Aufbrechen sozialer Differenzlinien wie Be-hinderung und Gender und die Förderung von Inklusion.

\subsection{Aufbrechen sozialer Differenzlinien}

Eine wesentliche Differenzlinie während der spielerischen Praktiken ist in Be-hinderungen vorzufinden. Es ist zu erkennen, dass Exklusionsmomente auftraten. Als Beispiel sind hier die Rahmenbedingungen wie Spielregeln, aber auch -materialien zu benennen. Dennoch gab es Versuche von den Spielenden, Exklusion zu verhindern. Hierbei konnten zwei unterschiedliche Herangehensweisen identifiziert werden.

Im Spielgeschehen wurden Ersatzhandlungen von Mitspielenden sichtbar, welche aber dazu führten, dass die von Exklusion betroffene Person keine eigene Ausführungs- bzw. Handlungsmacht besass. Das Angebot, Spielinteraktionen zu übernehmen, wurde entweder verbalisiert oder durch physische Interaktion, aber ohne verbalisierte Rücksprache direkt umgesetzt. In Variante 1 wurde die spielende Person als handlungsaktiv wahrgenommen, welche jedoch mittels Verniedlichungspraktiken oftmals als handlungsunfähig deklariert wurde (Goodley und RunswickCole 2010). Trotzdem wurden verbale Unterstützungsangebote in Rücksprache mit dem oder der Betroffenen besprochen. In Variante 2 hingegen wird der spielenden Person jegliche Handlungskompetenz abgesprochen, sodass ohne Nachfrage und Einverständnis Spielausführungen übernommen werden. Die Absprache von 
Handlungskompetenzen und die dadurch bedingte direkte Übernahme von Spielinteraktionen ist oftmals darauf zurückzuführen, dass spielerische Praktiken einen hohen kompetitiven Charakter aufweisen. Zudem wird das Spielgeschehen dadurch bestimmt, dass ein immer wiederkehrender Aushandlungsprozess zwischen den eigenen Bedürfnissen, Zielen und Möglichkeiten sowie den spielgemeinschaftlichen Interessen stattfindet. Anhand der vorgestellten Fallbeispiele lässt sich verdeutlichen, dass die direkte Handlungsausführung für eine spielende Person die Unterbrechung des Spielverlaufs verhindert und das Spiel effizient aufrechterhält. Somit können Zeit und Druck als Merkmale verzeichnet werden, welche dazu führen, dass Spielenden ihre Handlungsaktivität abgesprochen wird. Es lässt sich schlussfolgern, dass in spielerischer Praxis wenig Raum für individuelle Bearbeitungsmöglichkeiten zu finden sind, wenn ein hohes Mass an Zeitdruck und kompetitiven Einstellungen vorliegt.

Dem gegenüber stehen kooperative Unterstützungshandlungen von Mitspielenden, welche es von Exklusion bedrohten Spielenden ermöglichen, weiterhin Teil der Spielgemeinschaft zu sein. Die Betroffenen artikulieren ihre Bedürfnisse selbst und fordern gezielte Unterstützungsmassnahmen ein. Hierbei lässt sich erkennen, dass Handlungsaktivität zugeschrieben und gewährt wird, aber auch von der Person selbst wahrgenommen wird. Daran anschliessend lässt sich die Art und Weise der Kommunikation nennen. Die Analyse ergab, dass die Verwendung von schwerer und game-based Sprache Barrieren fördert, sodass nicht alle gleichberechtigt am Spiel teilnehmen können. Diesbezüglich muss aktiv beachtet werden, dass alle Teilnehmenden an Kommunikationsprozessen teilhaben können. Dieser Aspekt wird auch während der game-based dialogues erwähnt. In Bezug auf den schulbasierten Einsatz wurde Village Voices als einsteigendes Spiel für z. B. Schülerinnen und Schüler der Grundschule definiert (vgl. Yannakakis 2010). Die Schülerinnen und Schüler lernen dabei Konflikte zwischen der eigenen Bedürfnisbefriedigung und dem Gemeinwohl der Spielgemeinschaft zu lösen und diesem Spannungsverhältnis durch Kooperation und Kommunikation zu begegnen. Allerdings ergaben sich während des Spielens einige strukturelle Probleme bezüglich des Spieldesigns, da es viel Text zu lesen gibt und gerade Grundschüler und -schülerinnen erst mit dem Lesen beginnen (vgl. ebd.). Zusammenfassend lässt sich festhalten, dass kompetitive Spielpraktiken sowie Zeitressourcen und Druckmechanismen minimiert vorzufinden sind, sodass die Spielenden ausreichend Zeit und Ressourcen hatten, um auf individuelle Bedürfnisse sowie Anliegen der Gruppe einzugehen. Nichtsdestotrotz kann nicht von einem inklusiven Verständnis gesprochen werden. Normative Spielregeln und Rahmenbedingungen führen dazu, dass einzelne Spielende vom Ausschluss bedroht sind (vgl. Hinz 2002, 359). Nur durch Unterstützungsmassnahmen und Hilfsangebote ist es den Spielerinnen und Spielern möglich, sich dem normativen Leitbild anzupassen. 
Die zweite Differenzlinie ist durch die soziale Kategorie Gender vertreten. Der zentrale Punkt der Auswertung ist, dass in vielen Spieldesigns kein Augenmerk auf Gendersensibilisierung gelegt wird. Auch in Village Voices findet sich dieser Kritikpunkt wieder, da zunächst eine männliche Spielfigur erscheint und das Umstellen auf einen weiblichen Charakter nicht unmittelbar ersichtlich ist. Dies kann mit Miller und Summers (2007) bestätigt werden: Sie führen an, dass oftmals aufgrund fehlenden Bewusstseins nur männliche Spielfiguren designt werden (ebd., 733). Dieser unreflektierte Moment führt aber dazu, dass genderbezogene Stereotypen reproduziert werden, anstatt diese aufzubrechen.

Ein weiterer Erklärungsversuch ist aus sprachhistorischer Perspektive zu geben. Bothe und Schuh (2005) erläutern, dass sich die männliche Version über Jahrhunderte in unserem Sprachgebrauch konstituiert hat und sprachbezogenes Umdenken einen zeitlichen Faktor inhärent habe. Die Analyse ergab, dass es nach wie vor zur ausschliesslichen Verwendung der männlichen Version kommt. Allerdings ist ein gendersensibler Sprachgebrauch im empirischen Material vorzufinden. Durch direktes Ansprechen und Einfordern in Form von Verbesserungen ist zu erkennen, dass Lernprozesse stattfinden. Die Spielsituation provoziert somit Situationen, in denen zu einem gendersensiblen Sprachgebrauch angeregt wird, denn in darauffolgenden Interaktionen wird die weibliche Form kontinuierlich verwendet, wenn diese erforderlich ist. Allerdings bleibt dennoch die Frage offen, ob die neu erlernte Sensibilisierung habitualisiert wurde oder nur als Anpassungsmechanismus zu vermerken ist. Demgegenüber ist ein indirektes Verbesserungsverhalten zu erkennen, welches ebenfalls auf sprachlicher Ebene wirkt. Es lässt sich verzeichnen, dass eine gewisse Gendersensibilisierung im Sprachgebrauch bereits vorhanden ist, nur in dieser analysierten Situation nicht verwendet wurde. Der Unterschied zu einer direkten Verbesserung ist, dass die Person sich bekannte Strukturen ins Bewusstsein ruft und sich selbst revidierend ausbessert. Diese Verhaltensstrukturen können eindeutig als habitualisierende Praktiken verzeichnet werden. Jedoch wird immer noch ein externer Faktor (in diesem Fall ein Irritationsmoment durch andere Teilnehmende benötigt, um sich bekannte Strukturen zu vergegenwärtigen und alte zu revidieren. Nichtsdestotrotz sind hieran erste inklusive Prozesse zu erkennen.

\subsection{Förderung inklusionsbezogener Kernkompetenzen: Reflektierende Diskussion, Kreatives Denken und Konfliktlösungsstrategien}

Im Anschluss an die Definition der UNESCO (2005) soll jeder Person ein Zugang zu Bildung und gesellschaftlicher Teilhabe ermöglicht werden, sodass Partizipation auf allen Ebenen als übergeordnetes Ziel angestrebt wird. In dem Projekt eCrisis wurden drei Kompetenzen definiert, welche als Kernelemente für die Umsetzung dienen sollen: Reflektierende Diskussion, kreatives Denken und Konfliktlösungsstrategien. 
Die Reflektierende Diskussion erfolgte im Spielgeschehen vor dem Hintergrund der Verwendung von gendersensibler Sprache. Daran ist zu sehen, dass sich bereits sprachliche Veränderungen in Bezug auf die Etablierung eines gendersensiblen Sprachgebrauches im Anschluss an Bothe und Schuh (2005) herauskristallisieren, welche durch Reflexionsprozesse erfolgen. Zudem waren diese Personen sensibilisiert darauf, sodass sie andere Teilnehmende darauf aufmerksam machten, wenn diese keine Sensibilisierung vorwiesen. Spielerische Interaktionen eröffnen zudem einen Raum, um kreatives Denken zu fördern. Dabei zeigte sich, dass durch gemeinschaftliches kreatives Denken, Handlungsaktivitäten und deren aktive Umsetzung (vgl. Schmoelz 2017) Inklusion gefördert wird. Hierbei wurde analysiert, dass auftretende Probleme oder Konflikte als zentrales Moment verzeichnet werden können, um Lösungsstrategien kreativ zu entwickeln. Die Interaktion zeichnet sich durch ein kooperatives und kommunikatives Miteinander aus, welches durch ein wertschätzendes Miteinander geprägt ist. Anhand der Ergebnisse wird deutlich, dass die drei Kompetenzen eng miteinander verwoben sind, sich gegenseitig bedingen und gemeinsam inklusive Strukturen ermöglichen und fördern.

Subsummierend kann festgehalten werden, dass mittels reflektierender Diskussion, kreativem Denken und Konfliktlösungsstrategien als zentrale Kompetenzen Voraussetzungen und Grundbedingungen für inklusive Verhaltensmuster definiert werden können. Zum einen muss ein Verständnis für das Gegenüber vorhanden sein, welches auf Wertschätzung und Anerkennung basiert (vgl. Goodley und RunswickCole 2010). Zusätzlich muss ein Kooperations- und Kollaborationswille aller Spielenden vorliegen, damit eine angemessene Umsetzung erfolgen kann. In der post gamebased activity wird auch von den Spielenden angemerkt, dass die gespielten Spiele Kommunikation fördern und Kommunikation die Basis von Zusammenarbeit und gegenseitigem Achten sei. Ein Teilnehmer spricht an, dass das Spielen ihm ein Gefühl von Gemeinschaft vermittelt habe. Diese Aussage lässt sich mit McGonigal (2011) bestätigten: in unserer neoliberalen und individualisierten Gesellschaft tue sich kein bzw. nur sehr wenig Raum für Gemeinschaftlichkeit und Zusammenarbeit auf.

\section{Fazit}

Auf Basis der theoretischen und empirischen Erkenntnisse werden im Folgenden die Forschungsfragen noch einmal aufgegriffen. Die Spielenden werden bereits im Spielgeschehen mit verschiedenen Differenzlinien konfrontiert. Zum einen wurden externe Faktoren wie Spielregeln, -materialen, -design identifiziert, welche die Spielenden mit verschiedenen Differenzlinien konfrontierten. Dabei kann der Vorteil genannt werden, dass dies als Reflexionsfolie und Anschlusspunkt gesehen werden kann, um sozialen Differenzdimensionen aktiv zu begegnen. Als Nachteil kann definiert werden, dass externe Faktoren zur Reproduktion von Ungleichheit führen 
und diese durch passives Rezipieren unverändert bleiben. Zum anderen kann zwischenmenschliche Interaktion als Ausgangspunkt dafür gesehen werden, wie sozialen Differenzen begegnet wird. Es ist abhängig von habitualisierten Strukturen, welche Handlungsaktivitäten zugelassen bzw. angeboten werden. Kooperation, Kollaboration und Kommunikation sind drei wesentliche Merkmale, die inklusive Moment bedingen. Pointiert zusammengefasst bedeutet dies, dass Spiele selbst keinen direkten Einfluss auf die Förderung von Inklusion nehmen. Vielmehr werden in der sozialen Interaktion während des Spielens Empowermentstrategien und soziale Teilhabe gefördert. Ein weiteres zentrales Resultat war, dass das Potential, Inklusion zu fördern, in den post game-based activities zu sehen ist: hierbei kommt es zu einer reflektierenden Auseinandersetzung mit den Interaktionen, Ereignissen, aber auch persönlichen Gefühlen während des Spielgeschehens. Dieses festigt und visualisiert die gewonnen Erkenntnisse über die Auseinandersetzung mit sozialen Differenzen.

Alles in allem weist der Ansatz des game-based learning in Verbindung mit post game-based activities inklusionsfördernde Strukturen auf. Diese sind in der heutigen Zeit mehr denn je von Bedeutung, damit gesellschaftliche Transformation gelingt. Aktive, partizipative Teilhabe für alle wird forciert und die Bedrohung von Exklusion verhindert. Ein weiterer Aspekt, welcher in dieser Forschungsarbeit zum Tragen kommt, ist, dass Schülerinnen und Schülern alltagsnahe methodische und didaktische Tools zur Verfügung gestellt werden. Diese zielen auf eine Transformation des Schulalltags ab, welcher digital gestaltet werden müsste, damit Schülerinnen und Schüler lebensnahe Lern- und Bildungserfahrungen machen können.

Abschliessend ist zu erwähnen, dass in der Analyse weitere Differenzlinien aufgetreten sind, welche aufgrund der vorgeschriebenen Länge keinen Platz fanden. Migration und soziale Herkunft, aber auch Alter können genannt werden, um in weiteren Forschungsvorhaben integriert zu werden. Auf theoretischer Ebene könnte der Intersektionalitätsansatz verfolgt werden, um die verschiedenen Differenzlinien dekategorisierend zu betrachten.

\section{Literatur}

Biewer, Gottfried, Michelle Proyer, und Gertraud Kremsner. 2019. Inklusive Schule und Vielfalt. Stuttgart: Kohlhammer.

Bohnsack, Ralf. 2003. "Dokumentarische Methode und sozialwissenschaftliche Hermeneutik». Zeitschrift für Erziehungswissenschaft 6 (4): 550-571. https://doi.org/10.1007/s11618-0030057-7.

Bohnsack, Ralf, Bettina Fritzsche, und Monika Wagner Willi. 2014. Dokumentarische Video- und Filminterpretation. Methodologie und Forschungspraxis. Opladen: Barbara Budrich. 
Bothe, Alina, und Dominik Schuh. 2005. Geschlecht in der Geschichte - Integriert oder separiert? Gender als historische Forschungskategorie. Bielefeld: Transkript. https://doi.org/10.14361/ transcript.9783839425671.

Cheong, Yun-Gyung, Rilla Khaled, Christoffer Holmgård, und Georgios N. Yannakakis. 2015. «Serious Games for Teaching Conflict Resolution: Modeling Conflict Dynamics». In Conflict and Multimodal Communication, herausgegeben von Francesca D'Errico, Isabella Poggi, Alessandro Vinciarelli, und Laura Vincze, 449-75. Cham: Springer International Publishing. https://doi.org/10.1007/978-3-319-14081-0_21.

de Bono, Edward. 1970. Lateral thinking: creativity step by step. New York: Harper \& Row.

Dewey, John. 1933. Democracy and education. Buffalo: Prometheus Books.

Ebner, Martin, und Sandra Schön. 2011. "Lehrbuch für Lernen und Lehren mit Technologien». http://l3t.eu/homepage/das-buch/ebook.

eCrisis. 2018. «eCrisis». www.ecrisis.eu.

Europäische Kommission. 2016. «Active Inclusion». https://ec.europa.eu/social/main. jsp?catld=1059\&langld=en.

Fuchs, Peter. 2016. «Inklusion/Exklusion - theoretische Präzisierungen». In Handbuch für Inklusion und Sonderpädagogik, herausgegeben von Ingeborg Hedderich, Gottfried Biewer, Judith Hollenweger und Reinhard Makrowetz, 397-401. Bad Heilbrunn: Klinkhardt.

Gabriel, Sonja. 2007. «Teaching Human Rights with Video Games?»In The 11th European Conference on Game-Based Learning, herausgegeben von Maja Pivec und Joseph Gründer, 191196. Reading: Acadamic Conferences and Publishing International Limited.

Goodley, Dan, und Katherine Runswick-Cole. 2010. «Emancipating play. Dis/abled children, development and deconstruction». Disability \& Society 24 (4): 499-512. https://doi. org/10.1080/09687591003755914.

Hillmann, Karl-Heinz. 1994. Wörterbuch der Soziologie. Stuttgart: Körner.

Hinz, Andreas. 2002. «Von der Integration zur Inklusion - terminologisches Spiel oder konzeptionelle Weiterentwicklung?» Zeitschrift für Heilpädagogik 53: 354-361.

Husband, Charles. 2007. "Social Work in an Ethically Diverse Europe. The Shifting Challenges of Difference». Social Work and Society 5 (3): 1-18. https://ejournals.bib.uni-wuppertal.de/ index.php/sws/article/view/138

Iske, Stefan, und Dan Verständig. 2014. «Medienpädagogik und die Digitale Gesellschaft im Spannungsfeld von Regulierung und Teilhabe». Medienimpulse 4: 1-13. https://doi. org/10.21243/mi-04-14-07.

Le, Son, Peter Weber, und Marin Ebner. 2013. «Game-Based Learning. Spielend Lernen?»In L3T. Lehrbuch für Lernen und Lehren mit Technologien, herausgegeben von Martin Ebner und Sandra Schön. Zugriff am 29. Juni 2019 https://www.pedocs.de/volltexte/2013/8352/pdf/ L3T_2013_Le_Weber_Ebner_Game_Based_Learning.pdf

McGonigal, Jane. 2011. Realtiy is Broken. Why Games Make us Better and How They Can Change the World. New York: The Penguin Press. 
Michael, David, und Sande Chen. 2011. Serious games: Games that educate, train, and inform. Ohio: Course Technology.

Miller, Monika, und Alicia Summers. 2007. "Gender Differences in Video Game Characters' Roles, Appearances, and Attire as Portrayed in Video Game Magazines». Sex Roles 56 (7-8): 733-742. https://doi.org/10.1007/s11199-007-9307-0.

Miettinen, Reijo. 2000. «The concept of experiential learning and John Dewey's theory of reflective thought and action». International Journal of Lifelong Education 19 (1): 54-72. https://doi.org/10.1080/026013700293458.

Prensky, Marc. 2001. Digital Game-Based Learning. New York: McGraw-Hill.

Przyborski, Aglaja, und Wohlrab-Sahr. 2009. Qualitative Sozialforschung: Ein Arbeitsbuch. München: Oldenbourg Wissenschaftsverlag.

Schmoelz, Alexander. 2016. «Ernsthafte Spiele als Anlass für Ko-Kreativität?» In Game Based Learning. Dialogorientierung \& spielerisches Lernen analog und digital, herausgegeben von Johann Haag, Joseph Weißenböck, Wolfgang Gruber und Christian Freisleben-Teuscher, 107-118. Brunn am Gebirge: IKON.

Schmoelz, Alexander. 2017. "On Co-Creativity in Playful Classroom Activities». Creativity: Theories - Research - Applications 4 (1): 25-64. https://doi.org/10.1515/ctra-2017-0002.

Schmoelz, Alexander. 2018. «Enabling co-creativity through digital storytelling in education». Thinking Skills and Creativity 28: 1-13. https://doi.org/10.1016/j.tsc.2018.02.002.

Schmoelz, Alexander, Gertraud Kremsner, Michelle Proyer, Daniel Pfeiffer, Lisa-Katharina Möhlen, Kosta Karpouzis, und Gerogios Yannakakis. 2017. «Inklusiver Unterricht mit Digitalen Spielen». Medienimpulse 2: 1-15. https://doi.org/10.21243/mi-02-17-07.

Stenning, Keith, Alexander Schmoelz, Heather Wren, Elias Stouraitis, Theodore Scalsas, Constantine Alexopoulos, und Amelie Aichhorn. 2016. "Socratic dialogue as a teaching and research method for co-creativity?» Digital Culture \& Education 8 (2): 154-168. https://www. digitalcultureandeducation.com/volume-82-papers/socratic-dialogue-as-a-teaching-andresearch-method-for-co-creativity.

Ting-Toomey, Stella, und John Oetzel. 2001. Managing Intercultural Conflict Effectively. Thousand Oaks: CA.

UNESCO. 2005. «Guidelines for Inclusion. Ensuring Access to Education for All». Paris: UNESCO.

Voida, Amy, Sheelagh Carpendale, und Saul Greenberg. 2009. «The Mii and the Wii: Emphasizing the Individual and the Group in Console Gaming». Research Report 2009: 931-10, Department of Computer Science, University of Calgary, Calgary, Alberta T2N 1N4, Canada.

Waldschmidt, Anne. 2005. «Disability Studies: Individuelles, soziales und/oder kulturelles Modell von Behinderung?» Psychologie und Gesellschaftskritik 29 (1): 9-31. https://nbn-resolving.org/urn:nbn:de:0168-ssoar-18770.

Yannakakis, Georgios, Julian Togelius, Rilla Khaled, Arnav Jhala, Kostas Karpouzis, Ana Paiva, und Asimina Vasalou. 2010. Siren: Towards Adaptive Serious Games for Teaching Conflict Resolution. 4th European Conference on Games Based Learning, Copenhagen. http://www. image.ece.ntua.gr/papers/640.pdf. 